\title{
Association between cancer literacy and cancer-related behaviour: evidence from Ticino, Switzerland
}

\author{
Nicola Diviani, Peter J. Schulz \\ Institute of Communication and Health, Università della Svizzera Italiana, Lugano, Switzerland
}

\section{Significance for public health}

From a public health perspective, our findings underscore the importance of adapting informational and educational communication interventions designed to improve cancer prevention and screening to different audiences, which could differ not only in their functional health literacy, and in particular to put into place strategies to evaluate their success, including the actual transformation of information in relevant knowledge that can be used as a basis for health decision making. Moreover, the cancer literacy score is able to provide researchers and public health officials with detailed information on which are the main gaps in cancer knowledge and to identify the segments of the population that are more at risk. This information could be used to design and develop targeted informational campaigns for low health literate citizens.

\section{Abstract}

Background. This paper details the role of different dimensions of health literacy in the relationship between health literacy and cancerrelated health behaviours. In particular, Cancer Literacy is studied as an exemplar of a dimension of health literacy beyond basic reading and writing skills. The link between functional health literacy, Cancer Literacy and cancer-related health behaviours is investigated in a sample of Ticino (Switzerland) residents $(n=639)$.

Design and methods. Detailed data is collected about respondents' functional health literacy, Cancer Literacy, cancer information seeking behaviour, engagement in cancer preventive behaviours, participation to cancer screenings, and intention to adhere to current screening recommendations.

Results. Results confirm the added value of Cancer Literacy - compared to functional health literacy - in explaining people's cancer information seeking behaviour, their participation to several cancer screenings and their screening intention, underscoring the need to take into account dimensions of health literacy beyond basic functional skills.

Conclusions. From a public health perspective, findings provide further evidence on the importance of adapting informational and educational communication intervention designed to improve cancer prevention and screening to different audiences.

\section{Introduction}

Health literacy has been defined as the degree to which individuals have the capacity to obtain, process, and understand basic health information and services needed to make appropriate health decisions. ${ }^{1}$ The concept has gained widespread acceptance, ${ }^{2}$ because of the evidence of a positive relationship between health literacy and health-related behaviours. ${ }^{3-5}$ One of the main limitations of research on the relationship between health literacy and behaviour resides in the fact that it relies on measures of functional health literacy $(F H L)$, or people's ability to read and understand medical information, ${ }^{6}$ which does not fully capture the complexity of the concept, and could thus let existing literacy gaps undetected. ${ }^{7}$ Even though a large number of empirical studies has been devoted to assess the role of $F H L$, less is known about the role played by other dimensions of health literacy in explaining health behaviour. ${ }^{2,6,8-11}$

The existence of several dimensions of health literacy has been widely acknowledged. ${ }^{8,11}$ Most definitions underscore the important role not only of the simple mechanical acquisition of information, but also of other dimensions, such as the ability to process and transform it into relevant knowledge, which should therefore be taken into account. ${ }^{1,6,12}$ Nutbeam, ${ }^{6}$ for instance, distinguishes between three different dimensions of health literacy. A first dimension, $F H L$, refers to basic cognitive abilities (including reading and writing skills) enabling the individuals to understand relevant health information in everyday situations. A second dimension, interactive health literacy, encompasses more advanced cognitive and social skills enabling the individuals to undertake an active role within their social environment regarding their own health. A last dimension, critical health literacy, includes even more advanced cognitive abilities enabling the individuals to critically appraise health-related information and advice and to take appropriate health decisions. Schulz and Nakamoto also propose a three-tiered conceptualization of health literacy, stressing the importance of knowledge. ${ }^{12}$ In addition to basic reading and numeracy skills, their multidimensional concept of health literacy comprises declarative knowledge (factual knowledge related to health issues to be able to learn how to approach a health condition) and procedural knowledge (know-how to apply factual knowledge and use health information in a specific context) and judgment skills (the ability to judge on the basis of factual knowledge necessary to deal with novel situations).

Since a clear picture of the casual pathways linking health literacy and health-related behaviours is still missing, a deeper understanding of the role played by different dimensions of the concept in this process would be greatly beneficial to the advancement of research in this field. ${ }^{4,13,14}$

\section{Cancer literacy}

The main aim of this paper is to gain insights on the role played by a dimension of health literacy other than the functional one in explaining health behaviours. One of the main obstacles to research taking into account different dimensions is the fact that these have been theorized, but only rarely operationalized. One example of operational definition is the concept of Cancer Literacy $(C L)$, which has been defined as all the knowledge a layperson needs to possess to understand the information and advice the health system has to offer with regard to preventing, diagnosing and treating cancer. ${ }^{15}$ The operationalized dimensions are those of declarative and procedural knowl- 
edge about cancer, as conceptualized by Schulz and Nakamoto. ${ }^{12}$ The process of operationalization and validation of the measure of $C L$ provides evidence of the fact that the concept actually captures components of health literacy other than $F H L .{ }^{15}$ According to the authors of the measure, giving a correct answer to a specific pool of cancer knowledge questions also reflects people's interactive health literacy (e.g., ability to find and interpret cancer information of varying levels of difficulty), and their critical and judgmental skills (e.g., ability to assess appropriateness of information located). ${ }^{16}$

\section{Research question and hypotheses}

The importance of people's health literacy in explaining a wide range of cancer-related behaviours has been widely recognized. ${ }^{17-24}$ However, most of these studies have assessed health literacy using measures of $F H L$ only. We thus decided to study $C L$ as an exemplar of a dimension of health literacy encompassing more than basic reading and writing skills, and to investigate whether a measure that is specific to the field of cancer prevention could contribute to a deeper understanding of the link between health literacy and cancer-related health behaviours.

That said, our leading research question is: What is the role of $C L$ in explaining cancer-related health behaviours? Two distinct but interrelated hypotheses were developed. First, since they are different dimensions of the same overarching concept, we expected $F H L$ and $C L$ to be associated (Hypothesis 1a). Moreover, based on the existing literature showing that health literacy is positively associated with being a female, being younger, having an higher educational level and having a personal history of disease, ${ }^{13,25,26}$ we expected to find the same associations for both FHL and CL (Hypothesis $1 b$ ).

Second, since $C L$ is a more complex and comprehensive dimension of health literacy explicitly conceptualized in the field of cancer prevention, ${ }^{15}$ and in light of the evidence on the association between health literacy and cancer-related behaviours, $3,4,17-19,21,26,27$ we expected $C L$ to present a stronger association with cancer information seeking and cancer-related health behaviours than FHL (Hypothesis 2).

The two hypotheses were tested in a sample of Ticino (Switzerland) residents, by collecting detailed data about their FHL, CL, cancer information seeking, and cancer-related health behaviours.

\section{Design and methods}

\section{Data collection and sample}

The cross-sectional data analysed in this study was collected face-toface in an opportunity quota sample of 639 Ticino residents by a team composed by undergraduate and graduate communication students. Interviewers were systematically trained to ensure knowledge of the questionnaire and of the criteria for the recruitment of the respondents. Interviewers were instructed to conduct 10-20 interviews in their extended social circle, including respondents evenly distributed as regards gender, age, and education. Interviewers could interview only one member of their own nuclear family (parents and siblings) and only one person per household. To ensure representation of people with personal experience with cancer, each interviewer was required to include at least one person who had already received a cancer diagnosis.

\section{Measures}

\section{Functional health literacy}

Respondents' FHL was assessed by means of an Italian translation of the screening questions developed and validated by Chew and colleagues. ${ }^{28,29}$ To assess the applicability of the screening questions to the Swiss health system, they were informally discussed with a group of healthcare providers, who agreed in suggesting not to use the question How confident are you filling out medical forms by yourself? because this is not common practice in Swiss hospitals. The questions were also informally pretested using the think-aloud technique among a group of Italian-speaking Swiss citizens, ${ }^{30}$ who confirmed what had been pointed out by the healthcare providers. For this reasons only the two questions How often do you have someone help you read hospital materials? and How often do you have problems learning about your medical condition because of difficulty understanding written information? were included in the questionnaire.

\section{Cancer literacy}

Cancer literacy was assessed using the Cancer Literacy Score (CLS), an index formed by 37 knowledge items regarding different aspects of cancer, ${ }^{15}$ grouped in 5 subscales (cancer risk, detection and diagnosis, treatment, coping, and information). The measure has been shown to present acceptable overall internal consistency, test-retest reliability and construct validity, and is a continuous variable ranging from 0 to 100 , reflecting the percentage of correct answers. ${ }^{16}$

\section{Cancer-related health behaviours}

Four different cancer-related health behaviours were assessed. The first was cancer information seeking: respondents were asked if they had ever searched for cancer information. As it has been done in the past, ${ }^{31,32}$ people answering yes, once or yes, several times were categorized as cancer information seekers and people answering no as cancer information non-seekers. Second, participants were asked to provide details about their engagement in four behaviours known for being associated with diminished cancer risk: not smoking, eating fruits and vegetables, exercising, and using a sunscreen. For analytical purposes an index was created by assigning a value of 1 for a healthier answer and a 0 for a less healthy answer, and by summing the values of these four items. Third, past cancer screening participation was assessed by seven questions asking respondents whether they had ever had a colonoscopy or endoscopy, a FOBT (Fecal Occult Blood Test), a skin exam, a PSA (Prostate-Specific Antigen) exam, a rectal exam, a Pap test, or a mammography. The choice of the screenings was related to the Swiss Cancer League screening guidelines (www.cancerleague.ch). Lastly, in order not to take into account only actual behaviors, all respondents, irrespectively of screening history, gender and age, were asked to rate on a 7-point Likert scale their intention to adhere to the current screening recommendations.

\section{Control variables}

The associations between $F H L, C L$ and cancer-related health behaviours might be an artefact of the effect of some well-known common predictors, such as gender, age, educational level, personal cancer history, and having played an active role in the care of a third person suffering from cancer. ${ }^{13,25,26}$ Data about these aspects were thus collected and used to highlight group differences and as control variables in multivariate analysis.

\section{Statistical Analysis}

Basic frequency analyses were used to describe the characteristics of the whole sample. Hierarchical multivariate regression models were used to estimate the independent relationships between respondents' characteristics, FHL, CL and cancer-related behaviours (controlling for all covariates). All analyses were performed on the whole sample, except those aiming at investigating past screening behaviour, which were conducted on separate sub-samples including only respondents belonging to the target group for each of the different screening test. In order to take into account a slight overrepresentation of people below 30 years and of those with a secondary education in the sample and to be representative of the Ticino population estimates, ${ }^{33}$ data were weighted for education and age. 


\section{Results}

\section{Sample characteristics}

Slightly more than half of the sample (53.8\%) was female. Fifteen percent of the respondents were less than 30 years old, $22.5 \%$ were 30 $44,33.2 \%$ were $45-64$, and $29.5 \%$ were over 65 . The mean age of the sample was 51 years ( $\mathrm{SD}=18.4$ years). About $68 \%$ of the respondents were Swiss, non-Swiss respondents had been living in Switzerland for 26.6 years on average $(\mathrm{SD}=19.3)$. Forty-one percent of the sample had only completed compulsory education ( 9 years), $46.2 \%$ had a secondary school degree (e.g. high school, professional school), and 13.1\% had a college degree or higher. Eleven percent of the respondents reported having ever been diagnosed with cancer, and 33.8\% reported having played an active role in the cancer experience of a third person, and were thus labelled as caregivers.

\section{Functional health literacy}

In the first screening question for $F H L$ - i.e. how often one needs help reading hospital materials - the majority of the respondents (42\%) reported never needing help, one quarter (25\%) occasionally, $16 \%$ sometimes, $8.6 \%$ often, and very few of them always. Multivariate analysis showed that, controlling for all covariates, only educational level was significantly associated $(B=0.314, P<0.001, \beta=0.191)$ with how often people need help (Model $\mathrm{R}^{2}=0.064$ ).

The second screening question - i.e. how often one has problems learning about his/her medical condition because of difficulties reading written materials - yielded similar results. The majority of the respondents (35.2\%) reported that it never happens to them, slightly more than one third (31.9\%) occasionally, around $20 \%$ sometimes, $6.4 \%$ often, and very few of them that it happens all the time (3.4\%). Multivariate regression showed that not only educational level $(B=0.199, P<0.01, \beta=0.127)$, but gender $(B=-0.191, P<0.05, \beta=-0.089)$ and having a personal history of cancer $(B=-0.265, P<0.05, \beta=-0.078)$ as well were significantly associated with having problems learning about one's medical condition (Model $\mathrm{R}^{2}=0.033$ ). The two questions showed acceptable internal consistency (Cronbach's alpha $=0.727$ ) and were thus merged into a single index which was used in further analyses.

\section{Cancer literacy}

The CL scale presented satisfactory internal consistency (Cronbach's alpha $=0.769)$. Respondents scored on average $51.04(\mathrm{SD}=14.49)$ on the scale, meaning they answered correctly on average to around $50 \%$ of the knowledge items. Multivariate analysis showed that, controlling for all the other covariates, only gender $(B=5.531, P<0.001, \beta=0.191)$, educational level $(B=0.953, P<0.001, \beta=0.112)$, and caregiver status $(\mathrm{B}=7.353, \mathrm{P}<0.001, \beta=0.241)$ were significantly associated with $C L$ (Model $\mathrm{R}^{2}=0.114$ ). As hypothesized, when added to the model, both the screening questions for $F H L$ were shown to be significantly associated with $C L$ (Help read: $\mathrm{B}=2.269, \mathrm{P}<0.001, \beta=0.176$; Problems understanding: $\mathrm{B}=2.036, \mathrm{P}<0.001, \beta=0.150)$.

\section{Cancer-related health behaviours}

\section{Cancer information seeking}

Almost forty percent of the respondents answered positively when asked whether they had ever searched for cancer-related information. A logistic regression showed that, controlling for covariates, educational level $(\mathrm{P}<0.001)$, personal cancer history $(\mathrm{P}<0.001)$, and caregiver status $(\mathrm{P}<0.001)$ were significantly associated with cancer information-seeking (Table 1, Model 1 for details). As shown in Table 2 (Model 2), when $F H L$ was added to the baseline logistic model, no significant effect was found. On the other hand, when $C L$ was added to the model in a subsequent step, it was shown to be significantly associated with of cancer information seeking $(\mathrm{P}<0.001$, Table 1, Model 3 for details).

\section{Preventive behaviours}

Respondents reported engaging on average in $2.3(\mathrm{SD}=0.87$ ) behaviours out of the 4 included in the preventive behaviour index. A multivariate analysis highlighted a significant independent effect of educa-

Table 1. Hierarchical logistic regression analysis with cancer information seeking as dependent variable, predicted by respondents' characteristics, functional health literacy and cancer literacy $(n=639)$.

\begin{tabular}{|c|c|c|c|c|c|c|}
\hline \multirow[t]{2}{*}{ Variable } & \multicolumn{4}{|c|}{ Cancer information seeking } & \multicolumn{2}{|c|}{ Model $R^{2}$} \\
\hline & B & SE & Wald & $\operatorname{Exp}(B)$ & Cox and Snell & Nagelkerke \\
\hline \multicolumn{7}{|l|}{ Model 1} \\
\hline Gender & 0.125 & 0.191 & 0.427 & 1.133 & 0.187 & 0.255 \\
\hline Age & $-0.011^{*}$ & 0.006 & 3.827 & 0.989 & & \\
\hline Educational level & $0.588 * * *$ & 0.153 & 14.824 & 1.801 & & \\
\hline Cancer history & $1.807^{* * *}$ & 0.308 & 34.525 & 6.093 & & \\
\hline Caregiver & $1.636^{* * *}$ & 0.202 & 65.358 & 5.133 & & \\
\hline \multicolumn{7}{|l|}{ Model 2} \\
\hline Gender & 0.159 & 0.193 & 0.676 & 1.172 & 0.191 & 0.259 \\
\hline Age & $-0.012 *$ & 0.006 & 4.032 & 0.988 & & \\
\hline Educational level & $0.554 * * *$ & 0.155 & 12.800 & 1.739 & & \\
\hline Cancer history & $1.861^{* * *}$ & 0.311 & 35.770 & 6.428 & & \\
\hline Caregiver & $1.629 * * *$ & 0.203 & 64.654 & 5.098 & & \\
\hline Functional health literacy & 0.162 & 0.104 & 2.399 & 1.175 & & \\
\hline \multicolumn{7}{|l|}{ Model 3} \\
\hline Gender & -0.222 & 0.210 & 1.113 & 0.801 & 0.266 & 0.361 \\
\hline Age & $-0.017 * * *$ & 0.006 & 6.768 & 0.984 & & \\
\hline Educational level & $0.339 * *$ & 0.161 & 4.409 & 1.404 & & \\
\hline Cancer history & $2.032^{* * *}$ & 0.341 & 35.532 & 7.633 & & \\
\hline Caregiver & $1.455^{* * *}$ & 0.215 & 45.924 & 4.285 & & \\
\hline Functional health literacy & 0.042 & 0.113 & 0.135 & 1.043 & & \\
\hline Cancer literacy & $0.060 * * *$ & 0.009 & 50.030 & 1.062 & & \\
\hline
\end{tabular}


tional level only ( $\mathrm{B}=0.115, \mathrm{P}<0.05, \beta=0.092)$ on the preventive behaviour index. When $F H L$ and $C L$ were added to the baseline model, no significant effect of either variable was found.

\section{Past screening participation}

More than half of the respondents (54.7\%) reported having been screened for cancer. Multivariate logistic regressions were performed in selected subsamples (corresponding to the target groups of the different screening test) to investigate the association between respondents' characteristics, FHL and $C L$ and past participation to cancer screening. Different personal characteristics were associated with participation to the different screening tests: among female respondents aged $50+$, older respondents $(B=-0.110, P<0.001, \operatorname{Exp}(B)=0.896)$ and those with an higher education level $(\mathrm{B}=-0.942, \quad \mathrm{P}<0.05$, $\operatorname{Exp}(B)=0.390$ ) were significantly less likely to have been screened for breast cancer, among male respondents aged $50+$, older respondents were significantly less likely to have been screened for prostate cancer $(\mathrm{B}=-0.047, \mathrm{P}<0.05, \operatorname{Exp}(\mathrm{B})=0.954)$ and, in the whole sample, caregiver of a cancer patient were significantly more likely to have been screened for skin cancer $(B=-0.436, P<0.05, \operatorname{Exp}(B)=0.646)$. None of the personal characteristics taken into consideration were shown to be independently associated with cervix cancer and colon cancer screening. When $F H L$ was added to the models, it was shown not to be significantly associated to completion of any of the screening tests. $C L$, on the contrary, was shown to be significantly associated to participation to all the screenings but the one for prostate cancer (Skin: $\mathrm{B}=0.034, \mathrm{P}<0.001$, $\operatorname{Exp}(\mathrm{B})=1.034 ;$ Cervix: $\mathrm{B}=0.035, \mathrm{P}<0.001, \operatorname{Exp}(\mathrm{B})=1.035 ;$ Colon: $\mathrm{B}=0.076, \quad \mathrm{P}<0.001, \quad \operatorname{Exp}(\mathrm{B})=1.079 ;$ Breast: $\mathrm{B}=0.033, \quad \mathrm{P}<0.05$, $\operatorname{Exp}(\mathrm{B})=1.033)$.

\section{Screening intention}

Respondents showed an overall moderate intention to adhere to current screening recommendations $(\mathrm{M}=4.84, \mathrm{SD}=1.99)$, the modal response being 7 , chosen by $29.3 \%$ of them. As shown in Table 2 (Model 1), a multiple regression highlighted significant independent effects for gender $(\mathrm{P}<0.001)$, age $(\mathrm{P}<0.001)$, and personal cancer history $(\mathrm{P}<0.05)$ on the intention to get screened. When FHL was added to the baseline regression model, no significant effect was found (Table 2, Model 2). However, when $C L$ was added to the model in a subsequent step, both $F H L$ and $C L$ were shown to be significantly associated with the respondents' intention to get screened $(\mathrm{P}<0.001$, Table 2 , Model 3 for details).

\section{Discussion}

Aim of this study was to get a deeper understanding of the role played by a dimension of health literacy other than basic functional skills in explaining cancer-related behaviours. The results will be discussed in light of the hypotheses derived from our literature review.

FHL and $C L$ have been shown to be correlated, thus confirming Hypothesis 1a. However, data showed that the two dimensions of health literacy are only partially associated with the same individual characteristics, thus partly disconfirming Hypothesis $1 b$. As expected, educational level and gender were associated with both $F H L$ and $C L$. However, having a personal history of cancer was significantly associated only with $F H L$ and being a caregiver only with $C L$. Contrarily to our hypothesis, at a multivariate level age was not found to be associated neither with $F H L$ nor with $C L$. This result could be interpreted as evidence of the fact that age should not be considered a predictor of literacy in itself: $C L$ could increase with age because the older people get, the more occasions they have had to get in contact with people suffering from cancer. As regards our second hypothesis (Hypothesis 2), data showed that $C L$ was independently positively associated with cancer information seeking, participation to cancer screening, and intention to undergo cancer screening, while $F H L$ was not. None of the two constructs, however, was found to be significantly associated with engagement in preventive behaviour. This could be explained by the fact that, unlike participation to cancer screening or intention to get screened, the preventive behaviours under investigation were not specific to cancer. There are indeed several personal or environmental factors other than $C L$, e.g. body image issues,${ }^{34}$ that could play an important role in the decision for example not to smoke or to eat fruit and vegetables every day. It has to be stressed that, even if $C L$ was shown to be associated with most of the behaviours under investigations, the strength of this association was in most cases very weak. As regards past screening participation, we advance two possible explanations for these results. First of all, most of the existing screening tests can be used for both preventive and diagnostic purposes. Our data do not allow to make a distinction between the two cases and part of the respondents reporting having completed one or more of the screening tests could have done so because of specific health problems and not for preventive reasons. In such a case it is plausible that $C L$ would play only a limited role in their decisions. Secondly, the tests considered are very different as regards for instance the barriers associated with completing them, their popularity, and the amount of promotion surrounding them. A very popular test conducted in regular medical consultations (such as the PSA test for men or the mammography for women) could therefore be more likely to be completed by people with low $C L$, whereas a test which is less widely known would require a more active involvement, and therefore a higher level of $C L$, in order to be completed.

\section{Limitations}

It has to be acknowledged that this study suffers from some measurement-related limitations. First of all it relies on an opportunity quota sample, which is not representative of the population and limits

Table 2. Hierarchical regression analysis with screening intention as dependent variable, predicted by respondents' characteristics, functional health literacy and cancer literacy $(n=639)$.

\begin{tabular}{|c|c|c|c|}
\hline \multirow[t]{2}{*}{ Variable } & \multicolumn{3}{|c|}{ Screening intention } \\
\hline & B & S̆E & $\beta$ \\
\hline \multicolumn{4}{|l|}{ Model 1} \\
\hline Gender & $0.513^{* * *}$ & 0.164 & 0.127 \\
\hline Age & $-0.017 * * *$ & 0.005 & -0.159 \\
\hline Educational level & 0.064 & 0.128 & 0.022 \\
\hline Cancer history & $0.523^{*}$ & 0.251 & 0.084 \\
\hline Caregiver & 0.254 & 0.172 & 0.060 \\
\hline Model $R^{2}$ & 0.048 & - & - \\
\hline \multicolumn{4}{|l|}{ Model 2} \\
\hline Gender & $0.483^{* * *}$ & 0.164 & -0.120 \\
\hline Age & $-0.017 * * *$ & 0.005 & -0.159 \\
\hline Educational level & 0.109 & 0.130 & 0.037 \\
\hline Cancer history & 0.474 & 0.251 & 0.076 \\
\hline Caregiver & 0.274 & 0.171 & 0.065 \\
\hline Functional health literacy & -0.185 & 0.085 & -0.088 \\
\hline Model $R^{2}$ & 0.056 & - & - \\
\hline \multicolumn{4}{|l|}{ Model 3} \\
\hline Gender & 0.224 & 0.160 & 0.056 \\
\hline Age & $-0.016 * * *$ & 0.005 & -0.152 \\
\hline Educational level & -0.051 & 0.125 & -0.017 \\
\hline Cancer history & 0.348 & 0.240 & 0.056 \\
\hline Caregiver & -0.038 & 0.169 & -0.009 \\
\hline Functional health literacy & $-0.291 * * *$ & 0.082 & -0.139 \\
\hline Cancer literacy & $0.044^{* * *}$ & 0.006 & 0.319 \\
\hline Model R2 & 0.142 & - & - \\
\hline
\end{tabular}


the generalizability of the results. Secondly, FHL was measured by two of the three self-report screening questions validated by Chew and colleagues. ${ }^{28,29}$ The reason behind the choice of screening questions over other established health literacy measures is mainly practical. To date no validated Italian versions of the commonly used health literacy measures, e.g. REALM or TOFHLA, exists. ${ }^{35,36}$ Even an informal validation of an Italian version of such instruments, as the one that was conducted with the screening questions for the purposes of the present research (see above), was not deemed feasible within the timeframe of the study. Nevertheless, each one of the three screening questions was proven by their authors to be a valid measure of functional health literacy and to be correlated with other health literacy measures. It has to be noted that the validity was assessed in the clinical setting and no conclusive evidence exists of their validity in a different context. Third, past screening participation was measured by single indicators not taking into account the motivation for completing the test (preventive $v s$. diagnostic). Fourth, our cancer information-seeking measure was dichotomous, and no distinction between modes of acquisition (e.g. seeking $v s$. scanning) or between sources used was made, limiting the depth of our examination. In particular, this measure did not allow us to get a full understanding of the process how individuals seek and evaluate health information and apply it to their healthcare decisions. Last, the cross-sectional nature of our study did not allow us to make any causal claims. Our results would therefore need to be tested in an experimental setting to have a clearer picture of the casual pathways linking health literacy and health behaviours.

\section{Conclusions}

Despite these limitations, this study offers an important perspective on the communication processes involved in the individuals' health decision making. Communication scholars can profit from a deeper understanding of the potential consequences of an unsuccessful search for information: indeed people's ability to read health information (FHL) does not seem to be crucial in itself in predicting behaviour, while a more important role is played by the extent to which this information has been internalized (people's $C L$ ). Moreover, the findings have important implications for our theoretical model of health literacy, providing evidence of a link between $C L$, cancer information-seeking and cancer-related health behaviours and stressing once more the need to go beyond considering (and measuring) health literacy as mere functional literacy skills and to take other dimensions of health literacy (e.g. knowledge) into account. 8,15

Correspondence: Nicola Diviani, Institute of Communication and Health, Università della Svizzera Italiana, via G. Buffi 6, 6900 Lugano, Switzerland. Tel.: +41.586.664.757 - Fax: +41.586.664.647.

E-mail: nicola.diviani@usi.ch

Key words: health literacy, cancer, health information, health behaviour.

Contributions: the authors contributed equally.

Conflict of interests: the authors declare no potential conflict of interests.

Received for publication: 2 July 2014.

Accepted for publication: 24 August 2014.

CC Copyright N. Diviani and P. J. Schulz et al., 2014

Licensee PAGEPress, Italy

Journal of Public Health Research 2014; 3:295

doi:10.4081/jphr.2014.295

This work is licensed under a Creative Commons Attribution NonCommercial 3.0 License (CC BY-NC 3.0).

\section{References}

1. US Department of Health and Human Services. Healthy people 2010: understanding and improving health. Washington DC: US Department of Health and Human Services; 2001.

2. Baker D. The meaning and the measure of health literacy. J Gen Intern Med 2006;21;878-83.

3. DeWalt D, Berkman N, Sheridan S, et al. Literacy and health outcomes. J Gen Intern Med 2004;19:1228-39.

4. Ishikawa H, Yano E. Patient health literacy and participation in the health-care process. Health Expect 2008;11:113-22.

5. Nielsen-Bohlman L, Institute of Medicine Committee on Health Literacy. Health literacy: a prescription to end confusion. Washington DC: National Academies Press; 2004.

6. Nutbeam D. Health literacy as a public health goal: a challenge for contemporary health education and communication strategies into the 21st century. Health Promot Int 2000;15:259.

7. Friedman D, Corwin S, Dominick G, Rose I. African American men's understanding and perceptions about prostate cancer: why multiple dimensions of health literacy are important in cancer communication. J Commun Health 2009;34:449-60.

8. Frisch AL, Camerini L, Diviani N, Schulz PJ. Defining and measuring health literacy: how can we profit from other literacy domains? Health Promot Int 2012;27:117-26.

9. Nutbeam D. Health promotion glossary. Health Promot Int 1998;13:349-64.

10. Nutbeam D. Defining and measuring health literacy: what can we learn from literacy studies? Int J Publ Health 2009;54:303-5.

11. Zarcadoolas C, Pleasant A, Greer DS. Understanding health literacy: an expanded model. Health Promot Int 2005;20:195-203.

12. Schulz P, Nakamoto K. Emerging themes in health literacy. Stud Commun Sci 2005;5:1-10.

13. Paasche-Orlow MK, Wolf MS. The causal pathways linking health literacy to health outcomes. Am J Health Behav 2007;31:19-26.

14. Shieh C, Mays R, McDaniel A, Yu J. Health literacy and its association with the use of information sources and with barriers to information seeking in clinic-based pregnant women. Health Care Women Int 2009;30:971-88.

15. Diviani N, Schulz PJ. What should laypersons know about cancer? Towards an operational definition of cancer literacy. Patient Educ Couns 2011;85, 487-492.

16. Diviani N, Schulz PJ. First insights on the validity of the concept of cancer literacy: a test in a sample of Ticino (Switzerland) residents. Patient Educ Couns 2012;87:152-9.

17. Anker AE, Reinhart AM, Feeley TH. Health information seeking: a review of measures and methods. Patient Educ Couns 2011;82;34654.

18. Davis T, Arnold C, Berkel H, et al. Knowledge and attitude on screening mammography among low-literate, low-income women. Cancer 1996;78:1912-20.

19. Halbert CH, Barg FK, Guerra CE, et al. Cultural, economic, and psychological predictors of colonoscopy in a national sample. J Gen Intern Med 2011;26:1311-6.

20. Kim SP, Knight SJ, Tomori C, et al. Health literacy and shared decision making for prostate cancer patients with low socioeconomic status. Cancer Invest 2001;19:684-91.

21. Lindau ST, Tomori C, Lyons T, et al. The association of health literacy with cervical cancer prevention knowledge and health behaviors in a multiethnic cohort of women. Am J Obstet Gynecol 2002;186;938-43.

22. Samantha Garbers M, Chiasson MA. Inadequate functional health literacy in Spanish as a barrier to cervical cancer screening among immigrant Latinas in new York city. Prevent Chron Dis 2004;1:A07. 
23. Vanderpool RC, Kornfeld J, Rutten LF, Squiers L. Cancer information-seeking experiences: the implications of Hispanic ethnicity and Spanish language. J Cancer Educ 2009;24:141-7.

24. von Wagner C, Semmler C, Good A, Wardle J. Health literacy and self-efficacy for participating in colorectal cancer screening: the role of information processing. Patient Educ Couns 2009;75:352-7.

25. Hawkins NA, Berkowitz Z, Peipins LA. What does the public know about preventing cancer? results from the health information national trends survey (HINTS). Health Educ Behav 2010;37:490503.

26. von Wagner C, Knight K, Steptoe A, Wardle J. Functional health literacy and health-promoting behaviour in a national sample of British adults. J Epidemiol Commun Health 2007;61:1086-90.

27. Warner D, Procaccino JD. Women seeking health information: distinguishing the web user. J Health Commun 2007;12:787-814.

28. Chew LD, Bradley KA, Boyko EJ. Brief questions to identify patients with inadequate health literacy. Fam Med 2004;36:588-94.

29. Chew L, Griffin J, Partin M, et al. Validation of screening questions for limited health literacy in a large VA outpatient population. J
Gen Intern Med 2008;23:561-6.

30. Campanelli P. Testing survey questions: new directions in cognitive interviewing. Bull Methodol Sociol 1997;55:5-17.

31. Ramanadhan S, Viswanath K. Health and the information nonseeker: a profile. Health Commun 2006;20:131-9.

32. Rutten L, Squiers L, Hesse B. Cancer-related information seeking: hints from the 2003 health information national trends survey (HINTS). J Health Commun 2006;11:147-56.

33. USTAT. Dati statistici: tabelle Ticino e Svizzera. 2009. Available form: http://www.ti.ch/DFE/USTAT/DATI_CANTONE/default.asp

34. Saltonstall R. Healthy bodies, social bodies: men's and women's concepts and practices of health in everyday life. Soc Sci Med 1993;36:7-14.

35. Davis T, Long S, Jackson R, et al. Rapid estimate of adult literacy in medicine: a shortened screening instrument. Fam Med 1993;25:391.

36. Parker RM, Baker DW, Williams MV, Nurss JR. The test of functional health literacy in adults. J Gen Intern Med 1995;10:537-41. 